\title{
Spherically Symmetric Gravity as a Completely Integrable System*
}

\author{
H.A. Kastrup ${ }^{\dagger}$ and T. Thiemann ${ }^{\ddagger}$ \\ Institute for Theoretical Physics, RWTH Aachen, \\ 52056 Aachen, FR Germany
}

Preprint PITHA 93-35, November 93

\begin{abstract}
It is shown - in Ashtekar's canonical framework of General Relativity - that spherically symmetric (Schwarzschild) gravity in 4 dimensional space-time constitutes a finite dimensional completely integrable system. Canonically conjugate observables for asymptotically flat space-times are masses as action variables and - surprisingly time variables as angle variables, each of which is associated with an asymptotic "end" of the Cauchy surfaces. The emergence of the time observable is a consequence of the Hamiltonian formulation and its subtleties concerning the slicing of space and time and is not in contradiction to Birkhoff's theorem. The results are of interest as to the concept of time in General Relativity. They can be formulated within the ADM formalism, too. Quantization of the system and the associated Schrödinger equation depend on the allowed spectrum of the masses.
\end{abstract}

${ }^{*}$ Work supported by the Graduierten-Programm of the DFG

${ }^{\dagger}$ E-mail address: kastrup @ thphys.physik.rwth-aachen.de

†Present address: Center for Gravitational Physics and Geometry, Pennsylvania State University, University Park, PA 16802-6300, USA; E-mail address: thiemann @ phys.psu.edu 


\section{Introduction}

Recently we have shown[1] (quoted as ref. I in the following) that Ashtekar's constraints in his Hamiltonian formulation of general relativity can be solved completely - classically and quantum mechanically as well - for spherically symmetric field configurations. As the two basic canonically conjugate observables - in the sense of Dirac - we identified the mass squared $P=4 \mathrm{~m}^{2}$ and a quantity called $Q$ which in geometrical metric terms takes the form (modulo constraints, see below)

$$
Q=\frac{1}{4 m} \int_{\Sigma} d x(1-2 m / R)^{-1} \sqrt{(d R / d x)^{2}-q_{x x}(1-2 m / R)},
$$

where $R=\sqrt{q_{\theta \theta}}$ and $q_{x x}$ are defined by the line element

$d s^{2}=-(N(x, t) d t)^{2}+q_{x x}(x, t)\left(d x+N^{x}(x, t) d t\right)^{2}+q_{\theta \theta}(t, x)\left(d \theta^{2}+\sin ^{2} \theta d \phi^{2}\right)$.

The (local) variable $x$ is assumed to coincide asymptotically $(x \rightarrow \infty)$ with the usual Euclidean radial variable $r$ (our notation in the present paper is generally the same as in ref. I).

For the Schwarzschild solution

$$
q_{\theta \theta}=x^{2}, \quad q_{x x}=(1-2 m / x)^{-1}
$$

we have $Q=0$. Remembering Birkhoff's theorem on the uniqueness of Schwarzschild's solution in the context of spherical symmetry[2] one wonders whether there are "observable" configurations which have $Q \neq 0$.

We have argued in ref. I that such configurations are possible in the Hamiltonian picture where the diffeomorphisms are to be generated by finite constraint functionals and cannot be implemented "by hand" as this is done in the mainly geometrical proof of Birkhoff's theorem in the conventional space-time picture. A related observation was already discussed by Ashtekar and Samuel[3] for asymptotically flat Bianchi models.

The possibility of a nonvanishing observable $Q$ within the Hamiltonian framework is associated with the subtleties of slicing the 4-dimensional pseudoriemannian manifold into space and time: A nonvanishing $Q$ requires a nonvanishing shift $N^{x}$ !

This last observation was explicitly discussed in ref. I, but we did not try to give a concrete physical interpretation of the quantity $Q$. It is the aim 
of the present paper to give such an interpretation and discuss some of its implications.

The main result is that the quantity

$$
T=8 m Q
$$

can be interpreted as a time variable canonically conjugate to the mass $m$ ! This interpretation does not only follow from the formal canonical conjugacy of $T$ with respect to the mass $m$ but especially from the relations

$$
d T / d t=\left\{T, H_{t o t}\right\}_{\bar{\Gamma}}=2 N^{(\infty)}(t), \quad H_{\text {red }}=\left.H_{t o t}\right|_{\bar{\Gamma}}=2 m N^{(\infty)}(t),
$$

where $H_{t o t}$ means the total Hamiltonian consisting of the (nonvanishing) constraints and the surface terms (see eq. (2.4) below), $\{. .$.$\} denotes the Poisson$ bracket, $H_{\text {red }}$ means the value of $H_{t o t}$ on the constraint surface $\bar{\Gamma}$ where the constraints vanish and $N^{(\infty)}(t)$ is the lapse function $N(t)$ in the spatially asymptotic region under consideration (there may be several of such regions each one having its own lapse function).

This means that both Hamiltonians generate time translations as symmetry transformations - in contrast to gauge transformation - in asymptotically flat regions. The dependence of the "observable" $T(t)$ on the "unobservable" asymptotic time parameter $t$ is determined by the gauge dependent quantity $N^{(\infty)}(t),\left(N^{(\infty)}=1\right.$ for space-times which are asymptotically Minkowski flat). The factor 2 in eqs. (1.5) is due to the normalization of the Hamiltonians adopted here.

Notice that the observable $T$ is a volume, not a surface quantity!

Our results mean that spherically symmetric gravity constitutes a completely integrable system with respect to its observables the mass $m$ being an action, the time $T$ an angle variable! This resembles the situation in $(2+1)$ gravity

The results can be translated into the ADM framework where one sees that $T$ is simply related to an exact Hamilton-Jacobi solution of the (classical) Wheeler-DeWitt eq. for spherical gravity.

Quantization of the $(1+1)$-dimensional canonical system formed by the observables $m$ and $T$ is straightforward, but depends on the spectrum of $m$, namely whether it covers the whole real axis or whether it is bounded from below. 


\section{The model}

We here collect the main elements of spherically symmetric gravity in terms of Ashtekar's variables and refer to ref. I for further details.

In the spherically symmetric case the basic canonical variables in Ashtekar's approach to quantum gravity, namely the connection coefficients $A_{a}^{i}(x)$ as configuration variables and the densitized triads $\tilde{E}_{i}^{a}(x), a=1,2,3, i=1,2,3$ as momentum variables, can be expressed by 6 functions $A_{I}(t, x)$, and $E^{I}(t, x), I=$ $1,2,3$, where the $E^{I}$ are real and the $A_{I}$ are complex. Here $t$ is a "time" variable and $\mathrm{x}$ is a (local) spatial variable which becomes the usual Euclidean radial variable $r$ at spatial infinity.

The metric $\left(q_{a b}\right)$ on the 3-dimensional (Cauchy) surfaces $\Sigma^{3}$ takes the form

$\left(q_{a b}\right)=\operatorname{diag}\left(\frac{E}{2 E^{1}}, E^{1}, E^{1} \sin ^{2} \theta\right), \operatorname{det}\left(q_{a b}\right)=\frac{1}{2} E^{1} E \sin ^{2} \theta, E=\left(E^{2}\right)^{2}+\left(E^{3}\right)^{2}$,

which shows that the variables $E^{1}$ and $E$ determine the sign and the degeneracies of the spatial metric.

Integrating the Einstein-Ashtekar action over the unit sphere in $\Sigma^{3}=S^{2} \times \Sigma$, where $\Sigma$ is an appropriate 1-dimensional manifold (see below), we get the following effective $(1+1)$-dimensional action for spherically symmetric gravity

$$
\begin{aligned}
S= & \int_{R} d t\left[\int_{\Sigma} d x\left(-i E^{I} \dot{A}_{I}\right)-H_{t o t}\right] \\
& \text { with the total Hamiltonian } \\
H_{t o t}= & \int_{\Sigma} d x\left(i \Lambda G-i N^{x}\left(V_{x}-A_{1} G\right)+N C\right)+Q_{r}+P_{A D M}+E_{A D}(
\end{aligned}
$$

where

$$
\begin{aligned}
G= & \left(E^{1}\right)^{\prime}+A_{2} E^{3}-A_{3} E^{2}: \\
& \text { Gauss constraint function; } \\
V_{x}= & B^{2} E^{3}-B^{3} E^{2}: \\
& \text { vector constraint function; } \\
C= & \left(B^{2} E^{2}+B^{3} E^{3}\right) E^{1}+\frac{1}{2} E B^{1}: \\
& \text { scalar constraint function; } \\
\left(B^{1}, B^{2}, B^{3}\right)= & \left(\frac{1}{2}\left(\left(A_{2}\right)^{2}+\left(A_{3}\right)^{2}-2\right), A_{3}^{\prime}+A_{1} A_{2},-A_{2}^{\prime}+A_{1} A_{3}\right):
\end{aligned}
$$




$$
\begin{aligned}
& \text { "magnetic" fields; } \\
\Lambda: & \text { Lagrange multiplier for the Gauss constraint; } \\
N^{x}: & \text { "shift" (see eq. }(1.2)) \\
& \text { and Lagrange multiplier for the vector constraint; } \\
N= & N / \sqrt{\left(E E^{1} / 2\right)}: \text { Lagrange multiplier for the } \\
& \text { scalar constraint, } N: \text { lapse (see eq. (1.2)); } \\
Q_{r}= & \left.-i \int_{\partial \Sigma} \Lambda E^{1}: \text { charge of the remaining } O(2) \text {-symmetEyl } 1\right) \\
P_{A D M}= & \int_{\partial \Sigma} i N^{x}\left(A_{2} E^{2}+\left(A_{3}-\sqrt{2}\right) E^{3}\right): \\
& \operatorname{ADM-momentum~in~x-direction;~} \\
E_{A D M}= & \int_{\partial \Sigma} N\left(A_{2} E^{3}-\left(A_{3}-\sqrt{2}\right) E^{2}\right) E^{1}: \text { ADM-energy. }
\end{aligned}
$$

As usual [5, 6] the surface terms arise from the requirement that the 3 constraint functionals

$\bar{G}[A, E ; \Lambda]=\int_{\Sigma} d x \Lambda G, \bar{V}_{x}\left[A, E ; N^{x}\right]=\int_{\Sigma} d x N^{x} V_{x}, \quad \bar{C}[A, E ; \underset{\sim}{\sim}]=\int_{\Sigma} d x \underset{\sim}{N} C$

are functionally differentiable with respect to $A_{I}$ and $E^{I}$. A dot means $d / d t$ and a prime $d / d x$.

The (equal "time") Poisson brackets are

$$
\left\{A_{I}(x), E^{J}(y)\right\}=i \delta_{I}^{J} \delta(x, y),\left\{A_{I}(x), A_{J}(y)\right\}=\left\{E^{I}(x), E^{J}(y)\right\}=0 .
$$

The normalization of the energy $E_{A D M}$ is such that $E_{A D M}=2 m N^{(\infty)}, N^{(\infty)}=$ $N(t, x \in \partial \Sigma)$ for the Schwarzschild mass $m$ (with Newton's constant $G=$ $1, c=1)$.

The Hamiltonian $H_{t o t}$ generates gauge transformations and motions:

$$
\delta A_{I}=\left\{A_{I}, H_{t o t}\right\} \epsilon, \quad \delta E^{I}=\left\{E^{I}, H_{t o t}\right\} \epsilon
$$

where $\epsilon$ is a corresponding infinitesimal parameter. Explicitly we have

$$
\begin{aligned}
& \delta A_{1}=\left[\Lambda^{\prime}+\left(N^{x} A_{1}\right)^{\prime}+i \underset{\sim}{\sim}\left(B^{2} E^{2}+B^{3} E^{3}\right)\right] \epsilon, \\
& \delta A_{2}=\left[\Lambda A_{3}+N^{x} A_{2}^{\prime}+i \underset{\sim}{\sim}\left(B^{2} E^{1}+B^{1} E^{2}\right)\right] \epsilon,
\end{aligned}
$$




$$
\begin{aligned}
& \delta A_{3}=\left[-\Lambda A_{2}+N^{x} A_{3}^{\prime}+i \underset{\sim}{\sim}\left(B^{3} E^{1}+B^{1} E^{3}\right)\right] \epsilon \\
& \delta E^{1}=\left[N^{x}\left(E^{1}\right)^{\prime}-i \underset{\sim}{\sim}\left(A_{2} E^{2}+A_{3} E^{3}\right) E^{1}\right] \epsilon \\
& \delta E^{2}=\left[\Lambda E^{3}+\left(N^{x} E^{2}\right)^{\prime}-i \underset{\sim}{N}\left(A_{1} E^{1} E^{2}+\frac{1}{2} A_{2} E\right)-i\left(\underset{\sim}{\sim} E^{1} E^{3}\right)^{\prime}\right] \epsilon, \\
& \delta E^{3}=\left[-\Lambda E^{2}+\left(N^{x} E^{3}\right)^{\prime}-i \underset{\sim}{\sim}\left(A_{1} E^{1} E^{3}+\frac{1}{2} A_{3} E\right)+i\left(\underset{\sim}{\sim} E^{1} E^{2}\right)^{\prime}\right]
\end{aligned}
$$

Whereas the functions $E^{I}$ are real, the connection functions $A_{I}$ are complex, because

$$
A_{I}=\Gamma_{I}+i K_{I} \quad I=1,2,3
$$

where $\Gamma_{I}$ are the (reduced) coefficients of the spin connection and $K_{I}$ the corresponding coefficients of the extrinsic curvature. These coefficients can be expressed in terms of the functions $E^{I}$ :

$$
\left(\Gamma_{1}, \Gamma_{2}, \Gamma_{3}\right)=\left(-\beta^{\prime},-\left(E^{1}\right)^{\prime} \frac{E^{3}}{E},\left(E^{1}\right)^{\prime} \frac{E^{2}}{E}\right), \quad \beta^{\prime}=\frac{E^{2}\left(E^{3}\right)^{\prime}-E^{3}\left(E^{2}\right)^{\prime}}{E}
$$

where $\beta=\arctan \left(E^{3} / E^{2}\right)$, and

$$
\left(K_{1}, K_{2}, K_{3}\right)=\frac{1}{\sim E^{1}}\left(E ^ { 1 } \left(\dot{q}_{x x}-\left(q_{x x}\right)^{\prime} N^{x}-2 q_{x x}\left(N^{x}\right)^{\prime}, E^{2}\left(\dot{E}^{1}-N^{x}\left(E^{1}\right)^{\prime}\right) .\right.\right.
$$

\section{Topology of the Cauchy surfaces $\Sigma$ and asymp- totic properties at spatial infinity}

\subsection{Possible topologies}

For spherically symmetric systems the topology of the Cauchy 3-manifold $\Sigma^{3}$ is necessarily of the form $\Sigma^{3}=S^{2} \times \Sigma$ where $\Sigma$ is a 1-dimensional manifold. In this paper we are only interested in topological situations where $\Sigma$ is open or compact with boundary, especially when it is asymptotically flat: As was motivated already in ref. I we choose here

$$
\Sigma=\Sigma_{n}, \Sigma_{n} \cong K \cup \bigcup_{A=1}^{n} \Sigma_{A}
$$


i.e. the hypersurface is the union of a compact set $\mathrm{K}$ (diffeomorphic to a compact interval) and a collection of ends (each of which is diffeomorphic to the positive real line without the origin) i.e. asymptotic regions with outward orientation and all of them are joined to $\mathrm{K}$. This means, we have $\mathrm{n}$ positive real lines, including the origin, but one end of each line is common to all of them, i.e. these parts are identified. Since the identity map is smooth, this is still a $C^{\infty}$ (Hausdorff) manifold.

As an example consider the Kruskal-extended Schwarzschild-manifold (see e.g. ref.[7]), where we have two ends $\Sigma_{1}$ and $\Sigma_{2}$ each of which belongs to the asymptotic region $x \rightarrow \infty$ with $N_{2}^{(\infty)}=-N_{1}^{(\infty)}=-N^{(\infty)}$.

We want to point out here that the boundary of the compactum $\mathrm{K}$ has nothing to do with the location of a horizon, it is just a tool to glue the various ends together and thus is a kinematically fixed ingredient of the canonical formalism, whereas the location of a horizon will depend on the mass of the system which is a dynamical object. In particular, one and the same topological compactum will be used for all possible values of the mass. Thus, although it is appropriate to draw the spacetime pictures which one can find in textbooks for, say, the Schwarzschild configuration with parameter $m$, the lines $x=2 m$ which seperate the 4 Kruskal regions do not correspond to a specific coordinate value for the boundary of the compactum $\mathrm{K}$ and its time evolution. This property should not give rise to confusion because from the mainly geometrical point of view one is used to the fact that the topology of $\Sigma$ may change under evolution while in the Hamiltonian picture topology change is excluded by definition.

Notice that - according to eqs. (2.17)-(2.22) - we can have independent evolutions in different ends of $\Sigma$ by choosing the support of the Lagrange multipliers $\Lambda, N^{x}$ and $\underset{\sim}{\sim}$ appropriately.

\subsection{Asymptotic properties of the fields}

The following discussion of the asymptotic properties of the fields differs slightly from the one in ref. I. It is, however, more appropriate for the physical interpretation of the observables of the system discussed below.

Most important for our purpose are the asymptotically flat manifolds $\Sigma^{3}$, for 
which we have

$$
\lim _{r \rightarrow \infty} q_{a b}=q_{a b}^{0}+\frac{f_{a b}(\vec{x} / r, t)}{r}+O\left(1 / r^{2}\right),
$$

where $q_{a b}^{0}$ denotes a fixed flat Euclidean metric with coordinates $x^{a}$ and $r^{2}=$ $q_{a b}^{0} x^{a} x^{b}$.

In our case we have

$$
q_{r r} \rightarrow 1+q_{-1}^{r} / r+q_{-2}^{r} / r^{2} \ldots, \quad q_{\theta \theta} \rightarrow r^{2}+q_{1}^{\theta} r+q_{0}^{\theta} \ldots .
$$

We want to translate this asymptotic behaviour of the metric coefficients into that of the quantities $E^{I}$. This leads to the ansatz (compare eq. (2.1))

$$
\begin{aligned}
& E^{1} \rightarrow x^{2}+e_{1}^{1} x+e_{0}^{1}+\ldots \\
& E^{2} \rightarrow \sqrt{2} e_{1}^{2} x+e_{0}^{2}+e_{-1}^{2} / x+\ldots, \\
& E^{3} \rightarrow \sqrt{2} e_{1}^{3} x+e_{0}^{3}+e_{-1}^{3} / x \ldots,\left(e_{1}^{2}\right)^{2}+\left(e_{1}^{3}\right)^{2}=1 .
\end{aligned}
$$

These asymptotic relations have to be made compatible with those of the canonically conjugate quantities $A_{I}$, for which we start with the ansatz

$$
\begin{aligned}
& A_{1} \rightarrow a_{-1}^{1} / x+a_{-2}^{1} / x^{2}+\ldots, \\
& A_{2} \rightarrow a_{-1}^{2} / x+a_{-2}^{2} / x^{2}+\ldots, \\
& A_{3} \rightarrow \sqrt{2}+a_{-1}^{3} / x+a_{-2}^{3} / x^{2}+\ldots
\end{aligned}
$$

Because of the eqs. (2.23) and (2.24) the asymptotic properties of $E^{I}$ and $A_{I}$ are not independent. They imply the relations (recall that the $E^{I}$ are real!)

$$
\begin{gathered}
\Re e\left(a_{-1}^{1}\right)=0, \Re e\left(a_{-2}^{1}\right)=\frac{1}{\sqrt{2}}\left(e_{0}^{3} e_{1}^{2}-e_{0}^{2} e_{1}^{3}\right), \Re e\left(a_{-3}^{1}\right)=\sqrt{2} e_{-1}^{3}-e_{0}^{3} e_{0}^{2}, \\
0=e_{1}^{3}\left(\Rightarrow e_{1}^{2}=1\right), \Re e\left(a_{-1}^{2}\right)=-e_{0}^{3}, \Re e\left(a_{-2}^{2}\right)=-e_{-1}^{3}+e_{0}^{3}\left(\sqrt{2} e_{0}^{2}-\frac{1}{2} e_{1}^{1}\right), \\
\Re e\left(a_{-1}^{3}\right)=\frac{1}{\sqrt{2}} e_{1}^{1}-e_{0}^{2} .
\end{gathered}
$$

The eqs. (2.23) and (2.25) lead to identities if we express the time derivatives $\dot{q}_{x x}$ etc. by means of the evolution eqs. (2.17)-(2.22).

In order to fix the appropriate asymptotic behaviour of the quantities $E^{I}$ 
and $A_{I}$ we require

i) that - essentially following ref. [6] - the integrands of the Liouville form

$$
\Theta_{L}=-i \int_{\Sigma} E^{I} d A_{I}
$$

and the symplectic form

$$
\Omega=-i \int_{\Sigma} d E^{I} \wedge d A_{I}
$$

behave asymptotically as $O\left(1 / x^{2}\right)$ in order for the integrals to converge, and that

ii) the Gauss constraint function (2.5) vanishes at least as $O\left(1 / x^{2}\right)$ at spatial infinity. This is the only use we make of all the constraints as far as the asymptotic properties are concerned.

The condition $E^{I} d A_{I} \rightarrow O\left(1 / x^{2}\right)$ implies

$d a_{-1}^{1}=0, d\left(a_{-2}^{1}+\sqrt{2} a_{-1}^{2}\right)=0, e_{1}^{1} d a_{-2}^{1}+d a_{-3}^{1}+\sqrt{2} d a_{-2}^{2}+e_{0}^{2} d_{-1}^{2}+e_{0}^{3} d a_{-1}^{3}=0$,

and from $d E^{I} \wedge d A_{I} \rightarrow O\left(1 / x^{2}\right)$ we get

$$
d\left(\sqrt{2} a_{-2}^{1}+a_{-1}^{2}\right)=0, \quad d\left(e_{0}^{3}-\sqrt{2} a_{-2}^{1}\right)=0 .
$$

All the conditions obtained to far can be satisfied by requiring

$$
a_{-1}^{1}=0, \quad a_{-2}^{1}=0, \quad a_{-1}^{2}=0, \quad e_{0}^{3}=0, \quad a_{-3}^{1}+\sqrt{2} a_{-2}^{2}=0 .
$$

It will turn out that these conditions alone do not suffice to ensure the convergence properties of the quantities we are interested in. This will, however, be achieved by requiring the Gauss constraint function (2.5) to decrease at least as $O\left(1 / x^{2}\right)$ at spatial infinity. This implies the relations

$$
\begin{gathered}
e_{1}^{2}=1, \quad e_{1}^{1}-\sqrt{2} e_{0}^{2}=\sqrt{2} a_{-1}^{3},\left(\Rightarrow \Im m\left(a_{-1}^{3}\right)=0,\right. \text { compare eq. (3.12)) } \\
\sqrt{2} e_{-1}^{2}+e_{0}^{2} a_{-1}^{3}+\sqrt{2} a_{-2}^{3}=0
\end{gathered}
$$

The resulting expansions for $E^{I}$ and $A_{I}$ are compatible with the evolution eqs. (2.17)-(2.22) and the following asymptotic behaviour of the Lagrange multipiers:

$$
\Lambda \rightarrow O\left(1 / x^{2}\right), \quad N^{x} \rightarrow O\left(1 / x^{2}\right), \quad N^{(\infty)}=O(1), \Rightarrow \underset{\sim}{\sim} \rightarrow O\left(1 / x^{2}\right) .
$$


This asymptotic behaviour of the Langrange multipliers means that we allow for a spatially asymptotic $O(2)$ symmetry and for spatially asymptotic time translations - as opposed to the corresponding gauge transformations which require a stronger decrease for $\Lambda$ and $N$ (see ref. [1, 6]). In the following we shall not exploit the asymptotic $O(2)$ symmetry and shall treat it as a gauge symmetry. However, the possibility of generating time translations at spatial infinity is of utmost importance for the interpretation of the theory.

The asymptotic properties of $E^{I}$ and $A_{I}$ yield

$$
\begin{aligned}
E & \rightarrow 2 x^{2}\left(1+\sqrt{2} e_{0}^{2} / x+\left(\frac{1}{2}\left(e_{0}^{2}\right)^{2}+\sqrt{2} e_{-1}^{2}\right) / x^{2}+\ldots,\right. \\
A & \rightarrow 2\left(1+\sqrt{2} a_{-1}^{3} / x+\left(\sqrt{2} a_{-2}^{3}+\frac{1}{2}\left(a_{-1}^{3}\right)^{2}\right) / x^{2}+\ldots,\right. \\
B^{1} & \rightarrow \sqrt{2} a_{-1}^{3} / x+O\left(1 / x^{2}\right) \\
B^{2} & \rightarrow-a_{-1}^{3} / x^{2}+O\left(1 / x^{3}\right), \\
B^{3} & \rightarrow\left(3 a_{-3}^{2}+\sqrt{2} a_{-4}^{1}+a_{-3}^{1} a_{-1}^{3}\right) / x^{4}+O\left(1 / x^{5}\right) .
\end{aligned}
$$

The expansions for $E^{1}$ and $E$ imply

$$
q_{x x}=\frac{E}{2 E^{1}} \rightarrow 1+\left(\sqrt{2} e_{0}^{2}-e_{1}^{1}\right) / x+O\left(1 / x^{2}\right) .
$$

Comparing this expression with the asymptotic expansion of the Schwarzschild solution (1.3) we find

$$
\sqrt{2} e_{0}^{2}-e_{1}^{1}=2 m=-\sqrt{2} a_{-1}^{3} .
$$

The last equality follows from eq. (3.18).

It implies for the ADM-energy (2.13) that

$$
E_{A D M}=-\sqrt{2} N^{(\infty)} a_{-1}^{3}=2 m N^{(\infty)} .
$$

For a compact $\Sigma$ it is sufficient to require the fields and Lagrange multipliers to be smooth and finite everywhere. Obviously, the case of compact topologies is much more easier to handle from a technical point of view.

\section{Symplectic reduction of the model and its observables}

We first recall some basic facts from the theory of symplectic reduction (for further details see refs. [8, 9]). It was shown in ref. I that the present model is 
a field theory with first class constraints. Let $\Gamma, \bar{\Gamma}$ and $\hat{\Gamma}$ denote the full phase space, its constraint surface (where the constraints are satisfied identically) and its reduced phase space (i.e. the constraint surface, but points in it are identified provided they are gauge related). The (local) existence of the latter follows from general theorems that are valid for first class systems. Let

$$
\iota: \bar{\Gamma} \rightarrow \Gamma \text { and } \pi: \bar{\Gamma} \rightarrow \hat{\Gamma}
$$

denote the (local) imbedding into the large phase space and the projection onto the reduced phase space respectively. Call the symplectic structures on the 2 phase spaces $\Omega$ and $\hat{\Omega}$ respectively. Then the presymplectic structure on $\bar{\Gamma}$ is defined by the pull-backs

$$
\pi^{*} \hat{\Omega}:=\bar{\Omega}:=\iota^{*} \Omega .
$$

In practice one computes the constraint surface and thus obtains the imbedding. One then defines the presymplectic structure by the pull-back under the imbedding. After that one computes the gauge orbits and obtains the projection. The reduced symplectic structure is then defined by the pull-back under the projection.

On the other hand, if $\Theta$ and $\hat{\Theta}$ are the symplectic potentials (Liouville forms) for the symplectic structures, we obtain

$$
d \wedge\left(\iota^{*} \Theta-\pi^{*} \hat{\Theta}\right)=\iota^{*} \Omega-\pi^{*} \hat{\Omega}=0,
$$

whence $\iota^{*} \Theta-\pi^{*} \hat{\Theta}$ is (locally) exact:

$$
d S:=\iota^{*} \Theta-\pi^{*} \hat{\Theta} .
$$

Here $\mathrm{S}$ is the Hamilton-Jacobi functional which generates a singular canonical transformation from the large to the reduced phase space. Replacing the canonical momenta on $\Gamma$ by the functional derivatives of $S$ with respect to the canonical coordinates on $\Gamma$ solves the constraints because by doing so one pulls back the momenta to $\bar{\Gamma}$. Hence, one way of obtaining the reduced phase space is to solve the Hamilton-Jacobi equation for constrained systems. This has been done in ref. [9] and also in ref. I.

The relation (4.4) suggests an additional method : it says that, up to a total differential, one obtains the reduced symplectic potential simply by inserting the solution of the constraint equations into the full symplectic potential. 
For field theories there might also be boundary terms involved in this reduction process the contribution of which to the reduced symplectic structure does not vanish. They may be neglected at a first stage because they will be recovered when one checks whether the observables of the reduced phase space are finite and functionally differentiable.

It turns out that the last method is quite appropriate for our model.

In the following we only discuss the nondegenerate case $E^{1} E \neq 0$. It then follows that the vanishing of the scalar and vector constraint functions $C_{x}$ and $C$ (eqs. 2.6 and 2.7) is equivalent to the vanishing of the functions

$$
C_{2}=B^{2} E^{1}+\frac{1}{2} B^{1} E^{2}, \quad C_{3}=B^{3} E^{1}+\frac{1}{2} B^{1} E^{3},
$$

because

$$
E^{1} C_{x}=E^{3} C_{2}-E^{2} C_{3}, \quad C=E^{2} C_{2}+E^{3} C_{3} .
$$

The vanishing of $C_{2}$ and $C_{3}$ implies

$$
E^{2}=g B^{2}, E^{3}=g B^{3}, g=-2 \frac{E^{1}}{B^{1}} .
$$

Here we exclude the trivial case $B^{1}=0$ (see ref. I). For our purpose the following combinations will be of interest, too:

$$
\begin{aligned}
& A_{3} C_{2}-A_{2} C_{3}=E^{1}\left(B^{1}\right)^{\prime}+\frac{1}{2} B^{1}\left(\left(E^{1}\right)^{\prime}-G\right) \\
& A_{2} C_{2}+A_{3} C_{3}=A \gamma E^{1}+\frac{1}{2} B^{1}\left(A_{2} E^{2}+A_{3} E^{3}\right),
\end{aligned}
$$

where $\mathrm{G}$ is the Gauss constraint function (2.5) and

$$
A \equiv\left(A_{2}\right)^{2}+\left(A_{3}\right)^{2}, \quad \gamma \equiv A_{1}+\alpha^{\prime}=\frac{A_{2} B^{2}+A_{3} B^{3}}{A}
$$

We here assume $A \neq 0$, because otherwise $B^{1}=-1,\left(B^{1}\right)^{\prime}=0$ (eq. (2.8) and a vanishing expression (4.8) implies $\left(E^{1}\right)^{\prime}=0$ contradicting eq. (3.4). From eq. (4.8) we get the constraint

$$
K_{1} \equiv \frac{1}{2} B^{1}\left(E^{1}\right)^{\prime}+\left(B^{1}\right)^{\prime} E^{1}=0, \text { or }\left(E^{1}\right)^{\prime}=g\left(B^{1}\right)^{\prime} .
$$


Integration yields

$$
\sqrt{E^{1}} B^{1}=\text { const. . }
$$

From the eqs. (3.4), (3.23) and (3.27) we obtain

$$
\sqrt{E^{1}} B^{1}=-2 m
$$

This is our first observable. That this is so follows from

$$
\left.\left(\delta\left(\sqrt{E^{1}} B^{1}\right)\right)\right|_{\bar{\Gamma}}=\left.\left\{\left(\sqrt{E^{1}} B^{1}\right), H_{t o t}\right\}\right|_{\bar{\Gamma}}=0
$$

where the eqs. (2.18), (2,19), (2.20), (4.7) and (4.11) have been used.

For the further discussion it is convenient to make use of the $O(2)$-symmetry in the $(2,3)$-" plane" of the variables $\left(A_{2}, A_{3}\right)$ and $\left(E^{2}, E^{3}\right)$ by introducing cylindrical coordinates

$$
\left(A_{2}, A_{3}\right)=\sqrt{A}(\cos \alpha, \sin \alpha), \quad\left(E^{2}, E^{3}\right)=\sqrt{E}(\cos \beta, \sin \beta) .
$$

They implies the relations

$$
G=\left(E^{1}\right)^{\prime}-\sqrt{A E} \sin (\alpha-\beta)
$$

and

$$
E^{I} d A_{I}=\pi_{\gamma} d \gamma+\pi_{1} d B^{1}+\pi_{\alpha} d \alpha-\frac{d}{d x}\left(E^{1} d \alpha\right)
$$

where

$$
\pi_{\gamma}=E^{1}, \quad \pi_{1}=\sqrt{\frac{E}{A}} \cos (\alpha-\beta), \quad \pi_{\alpha}=G .
$$

We see that the change of variables is tantamount to a canonical transformation where one of the new momenta is the Gauss constraint function $G$ ! In addition, the r.h.s of eq. (4.9) now takes the form

$$
A\left(\pi_{\gamma} \gamma+\frac{1}{2} \pi_{1} B^{1}\right)
$$

implying the constraint

$$
K_{2} \equiv 2 \pi_{\gamma} \gamma+\pi_{1} B^{1}=0, \text { or } \pi_{1}=g \gamma
$$


Setting $G=0$ we get for the constraint functions $C$ and $V_{x}$ in terms of the new variables

$$
\begin{aligned}
C & =\left(1+B^{1}\right) \pi_{1} K_{2}+\frac{1}{2\left(1+B^{1}\right)} \pi_{\gamma}^{\prime} K_{1}, \\
V_{x} & =-\left(E^{1}\right)^{\prime} \gamma+\left(B^{1}\right)^{\prime} \pi_{1} .
\end{aligned}
$$

Dropping the last term in eq. (4.17) which leads to a surface integral to be ignored (see the remarks following eq. (4.4) above) the integrand of the Liouville form reduced with respect to the Gauss constraint is given by

$$
\pi_{\gamma} d \gamma+\pi_{1} d B^{1}=-\gamma d \pi_{\gamma}-B^{1} d \pi_{1}+d(\ldots) .
$$

Using the relation (4.20) we have

$$
\gamma d \pi_{\gamma}+B^{1} d \pi_{1}=\sqrt{\pi_{\gamma}} B^{1} d\left(\frac{\pi_{1}}{\sqrt{\pi_{\gamma}}}\right)
$$

so that the Liouville form (3.13) finally reduces to

$$
\Theta_{L}=-i \int_{\Sigma} E^{I} d A_{I}=i \int_{\Sigma} \sqrt{\pi_{\gamma}} B^{1} d\left(\frac{\pi_{1}}{\sqrt{\pi_{\gamma}}}\right)+d \hat{S}=m d T+d \hat{S}
$$

where eq. (4.13) has been used and where

$$
T=T\left[\pi_{1}, \pi_{\gamma}\right]=\int_{\Sigma} \lambda, \quad \lambda \equiv-2 i \frac{\pi_{1}}{\sqrt{\pi_{\gamma}}}
$$

The quantity $T$ is our second observable:

From eqs. (4.13) and (4.20) we have

$$
\lambda=-2 i g \frac{\gamma}{\sqrt{\pi_{\gamma}}}=4 i \frac{\sqrt{\pi_{\gamma}}}{B^{1}} \gamma=8 m \rho, \rho \equiv-i \frac{\gamma}{\left(B^{1}\right)^{2}}
$$

and since the asymptotic relations for $A_{2}, A_{3}, A, B^{2}, B^{3}$ and $B^{1}$ (eqs. (3.16), (3.18) and (3.22-3.25)) imply $\rho \rightarrow O\left(1 / x^{2}\right)$ the integral $T$ exists. From ref. I we know that $B^{1}$ is weakly real and $\gamma$ weakly imaginary. So $T$ is weakly real. Most important for our interpretation of $T$ is the relation

$$
\delta \rho=\frac{d}{d x}\left[N^{x} \rho+\frac{4 m^{2} \underset{N}{\sim}\left(B^{1}\right)^{\prime}}{A\left(B^{1}\right)^{4}}\right] \epsilon,
$$


which follows from applying eqs. (2.17)-(2.19) to

$$
\delta \gamma=\delta\left(A_{1}+\alpha^{\prime}\right), \quad \delta \alpha=\left(A_{2} \delta A_{3}-A_{3} \delta A_{2}\right) / A, \quad \delta B^{1}=\delta A / 2
$$

and using the relations (4.7) and (4.13). The expression in the square brackets of eq. (4.26) approaches the value $N^{(\infty)} /(4 m)$ for large $x$. Combining this with eqs. (4.14) and (4.25) we get the important result

$$
\delta T=\left\{T, H_{t o t}\right\} \epsilon=2 \sum_{A=1}^{n} N_{A}^{(\infty)} \epsilon
$$

where we have allowed for different lapses $N_{A}^{(\infty)}$ at different ends $\Sigma_{A}$ (see eq. (3.1)) and where we have assumed that there are no contributions from possible inner boundary points. The occurrence of the lapses on the r.h.s. of $\delta T$ is due to our allowance for time translations as a symmetry transformation at spatial infinity ([6]), as opposed to a gauge transformation for which $N^{(\infty)}=0$.

If spacetime is Minkowski-like at spatial infinity we have $N^{(\infty)}=1$ and - interpreting the parameter $\epsilon$ as the (infinitesimal) proper time of an asymptotic observer and considering one end only- we get

$$
\dot{T}=2, \quad T=2 t+\text { const. . }
$$

Thus, the observable $T$ is to be interpreted as a time variable! (The factor 2 is a consequence of our normalization of the energy - see eq. (3.28)).

Notice that eq. (4.26) and the eqs. (2.17)-(2.22) allow for different - even independent - evolutions of $T$ in different ends $\Sigma_{A}$ by choosing the supports of the Lagrangean multipliers $\Lambda, N^{x}$ and $N$ (or $N$ ) appropriately!

The mass $m$ is canonically conjugate to the time $T$ : Let us define the mass $M=m$ by

$$
M=-\frac{1}{2} \int_{\Sigma} \sqrt{\pi_{\gamma}} B^{1} \chi, \quad \int_{\Sigma} \chi=1,
$$

where $\chi$ is a suitable smooth test function the support of which can again be concentrated on a given end $\Sigma_{A}$. From eqs. (3.13) and (4.17) we infer the Poisson brackets

$$
\left\{B^{1}(x), \pi_{1}(y)\right\}=i \delta(x, y),\left\{\gamma(x), \pi_{\gamma}(y)\right\}=i \delta(x, y),\left\{\pi_{1}(x), \pi_{\gamma}(y)\right\}=0 \text { etc. }
$$


for the new canonical variables. We therefore have

$$
\begin{aligned}
\{T, M\} & =\int_{\Sigma} d x \int_{\Sigma} d y i\left\{\left(\frac{\pi_{1}}{\sqrt{\pi_{\gamma}}}\right)(x),\left(\sqrt{\pi_{\gamma}} B^{1}\right)(y) \chi(y)\right\} \\
& =\int_{\Sigma} d x \int_{\Sigma} d y i \sqrt{\frac{\pi_{\gamma}(y)}{\pi_{\gamma}(x)}}\left\{\pi_{1}(x), B^{1}(y)\right\} \chi(y)=\int_{\Sigma} d y \chi(y)=1 .
\end{aligned}
$$

We see that we can interpret spherical symmetric gravity as a $(1+1)$-dimensional completely integrable canonical system where the mass $M$ is the action and the time $T$ the angle variable.

This is in complete agreement with the structure of the reduced Hamiltonian $H_{\text {red }}=\left.H_{\text {tot }}\right|_{\bar{\Gamma}}$ which according to eqs. (2.4) and (3.28) takes the form

$$
H_{\text {red }}=2 M N^{(\infty)}
$$

because the constraints $G, C_{x}$ and $C$ vanish now and we have $P_{A D M}=0$ from our choice of the asymptotic properties of the fields and of $N^{x}$. In addition we have $Q_{r}=0$ by an appropriate choice of the decay $\left(O\left(1 / x^{2+\epsilon}\right)\right)$ of $\Lambda$. Eq. (4.31) then yields

$$
\dot{T}=\left\{T, H_{\text {red }}\right\}=2 N^{(\infty)},
$$

in complete agreement with eq. (4.27) above.

In deriving the results above the introduction of the variables $\gamma, \pi_{\gamma}$ and $\pi_{1}$ has been quite useful. The advantage of these canonical variables will be especially apparent when solving the Reissner-Nordstrøm model [10].

\section{Relations to the spacetime metric}

We first express $T$ as functional of the metric coefficients $q_{r r}$ and $q_{\theta \theta}$ : From eqs. (4.16) and (4.18) we get - without using any constraint -

$$
\pi_{1}^{2}=\frac{E}{A}-\frac{\left(\left(E^{1}\right)^{\prime}-G\right)^{2}}{A^{2}} .
$$

Assuming $G=0$, dividing by $E^{1}$ and taking the square root yields

$\lambda=2 \frac{1}{A \sqrt{E^{1}}}\left(\left(\left(E^{1}\right)^{\prime}\right)^{2}-A E\right)^{1 / 2}=2(1-2 m / R)^{-1}\left(\left(R^{\prime}\right)^{2}-q_{x x}(1-2 m / R)\right)^{1 / 2}$, 
where the relations

$$
q_{x x}=\frac{E}{2 E^{1}}, R=\sqrt{q_{\theta \theta}}=\sqrt{E^{1}}, A=2\left(1+B^{1}\right)=2\left(1-\frac{2 m}{R}\right)
$$

have been used. The difference $\left(\left(E^{1}\right)^{\prime}\right)^{2}-E A$ behaves as $O(1 / x)$ for large $x$ if the properties $(3.21),(3.22),(3.18)$ and $(3.19)$ hold. In that case the integral

$$
\begin{aligned}
T\left[q_{x x}, q_{\theta \theta}\right] & =\int_{\Sigma} 2(1-2 m / R)^{-1} w\left(q_{x x}, q_{\theta \theta}\right) \\
w\left(q_{x x}, q_{\theta \theta}\right) & =\left(\left(R^{\prime}\right)^{2}-q_{x x}(1-2 m / R)\right)^{1 / 2}
\end{aligned}
$$

exists because $w \rightarrow O\left(x^{-3 / 2}\right)$. Notice that the decay of the integrand of $T\left[q_{x x}, q_{\theta \theta}\right]$ is determined here without refering to the constraint (4.20) which we used in the context of eq. (4.25) when calculating the asymptotic behaviour of $\lambda$ there!

For the quantity $T$ to be real the inequality

$$
\left(R^{\prime}\right)^{2} \geq q_{x x}\left(1-\frac{2 m}{R}\right)
$$

has to be satisfied. As was already mentioned above this this is guaranteed due to the property of $T$ to be weakly real.

For the Schwarzschild solution (1.3) $T$ vanishes. How is such a vanishing $T$ to be reconciled with the eq. (4.27)? The answer lies in the fact that the Schwarzschild-Kruskal manifold has two ends for $x \rightarrow \infty$ with $N_{2}^{(\infty)}=$ $-N_{1}^{(\infty)}$ so that the sum on the r.h.s. of eq. (4.27) vanishes! It is amusing that here a second end is required in order to obtain consistency of the formalism! Nonvanishing values of $T$ will be discussed below.

The important eq.(4.27) can be derived in the ADM framework, too: It follows from eqs. (2.20)-(2.22) that

$$
\begin{aligned}
\delta R & =\left(N^{x} R^{\prime}+\underset{\sim}{\sim} R^{2} w\right) \epsilon \\
\delta q_{x x} & =\left[2 q_{x x}\left(N^{x}\right)^{\prime}+q_{x x}^{\prime} N^{x}+\frac{1}{w}\left(2 q_{x x} R^{2} R^{\prime \prime}-q_{x x}^{\prime} R^{2} R^{\prime}-2 m q_{x x}^{2}\right) \underset{\sim}{N}\right] \epsilon
\end{aligned}
$$

In obtaining the last equations the relations

$$
\delta R=\frac{1}{2 R} \delta E^{1}, \quad \delta q_{x x}=\frac{1}{2 R^{2}} \delta E-\frac{2 q_{x x}}{R} \delta R, \quad \delta E=2\left(E^{2} \delta E^{2}+E^{3} \delta E^{3}\right),
$$


have been used. For the derivation of eq. (5.6) the relations $E^{2}\left(E^{3}\right)^{\prime}-$ $E^{3}\left(E^{2}\right)^{\prime}=\beta^{\prime} E$ and

$$
\begin{aligned}
\beta^{\prime}= & \alpha^{\prime}-\left[\arccos \left(\frac{R^{\prime}}{\sqrt{q_{x x}\left(1-\frac{2 m}{R}\right)}}\right)\right]^{\prime}, \\
& \arccos z=-i \cosh ^{-1} z=-i \ln \left(z+\sqrt{z^{2}-1}\right), z \geq 1,
\end{aligned}
$$

are essential. The last one follows from eq. (4.16) with $G=0$ and observing that $(\arcsin z)^{\prime}=(\arccos z)^{\prime}$ for $z \geq 1$.

The rhs. of eq. (4.28) arises here from the term

$$
\frac{d}{d x}\left(\frac{\partial \lambda}{\partial R^{\prime}} \delta R=\frac{2 R^{\prime}}{w\left(1-\frac{2 m}{R}\right)} \delta R\right)
$$

which appears in the integrand when $T$ is varied with respect to $R$. Collecting all the terms we get

$$
\delta T\left[q_{x x}, R\right]=2 \int_{\partial \Sigma}\left(1-\frac{2 m}{R}\right)^{-1}\left(w N^{x}+R^{2} R^{\prime} \underset{\sim}{\sim}\right) \epsilon
$$

The first surface term vanishes and the second one gives eq. (4.28). Eq. (5.9) is equivalent to eq. (4.27) above or eq. (5.35) below.

The observable $T$ is related in a very simple way to the functional

$$
\begin{aligned}
S\left[q_{x x}, R ; m\right)= & \int_{\Sigma} u\left(q_{x x}, R, m\right) \\
u\left(q_{x x}, R, m\right)= & 2 R\left(R^{\prime} \cosh ^{-1}\left(\frac{R^{\prime}}{\sqrt{q_{x x}\left(1-\frac{2 m}{R}\right)}}\right)-w\right), \\
& \cosh ^{-1}\left(\frac{R^{\prime}}{\sqrt{q_{x x}\left(1-\frac{2 m}{R}\right)}}\right)=\ln \left(R^{\prime}+w\right)-\frac{1}{2} \ln \left(q_{x x}\left(1-\frac{2 m}{R}\right)\right),
\end{aligned}
$$

derived in ref. I, which provides an exact Hamilton-Jacobi solution of the (classical) Wheeler-DeWitt eq.

$$
\frac{1}{R^{2} \sqrt{q_{x x}}}\left[2 q_{x x}^{2} p_{x x}^{2}-2 q_{x x} R p_{x x} p_{R}+2 R^{2}\left(2 R R^{\prime \prime}+\left(R^{\prime}\right)^{2}-\frac{q_{x x}^{\prime} R R^{\prime}}{q_{x x}}-q_{x x}\right)\right]=0
$$


for spherical symmetric gravity [11], where $p_{x x}$ and $p_{R}$ are the canonical momenta conjugate to $q_{x x}$ and $R$, respectively (the radial function $R$ here should not be confused with the curvature scalar $R$ which does not appear in this paper at all!).

The integral $S$ exists because the integrand $u$ behaves as $u \rightarrow O\left(x^{-2}\right)$ if $w \rightarrow O\left(x^{-3 / 2}\right)$ for large $x$ as supposed above. Furthermore, since

$$
\frac{\partial u}{\partial R^{\prime}}=2 R\left[\ln \left(R^{\prime}+w\right)-\frac{1}{2} \ln \left(q_{x x}\left(1-\frac{2 m}{R}\right)\right)\right]
$$

the functional $S\left[R, q_{x x} ; m\right)$ is functionally differentiable with respect to $R$ if $\delta R \rightarrow O(1)$ for $x \rightarrow \infty$ because then the surface term

$$
\frac{\partial u}{\partial R^{\prime}} \delta R
$$

vanishes for $x \rightarrow \infty$ if $w \rightarrow O\left(x^{-3 / 2}\right)$.

Inserting

$$
\begin{aligned}
p_{x x} & =\frac{\delta S}{\delta q_{x x}}=\frac{\partial u}{\partial q_{x x}}=-\frac{w R}{q_{x x}} \\
p_{R} & =\frac{\delta S}{\delta R}=\frac{\partial u}{\partial R}-\frac{d}{d x}\left(\frac{\partial u}{\partial R^{\prime}}\right) \\
& =-2 w-\frac{1}{w}\left(2 R R^{\prime \prime}-\frac{q_{x x}^{\prime} R R^{\prime}}{q_{x x}}-\frac{2 m}{R} q_{x x}\right)
\end{aligned}
$$

into the Wheeler-DeWitt eq. (5.11) solves that equation identically. In the same way the ADM diffeomorphism constraint [1]

$$
\frac{1}{q_{x x}}\left(q_{x x} p_{x x}-\left(2 q_{x x} p_{x x}\right)^{\prime}+R^{\prime} p_{R}\right)=0
$$

is fulfilled. The solution $S\left(q_{x x}, R\right)$ was also discussed in ref. [12]. The quantities $S$ and $T$ are related as follows: Eq. (4.24) gives

$$
\Theta_{L}=-T d m+d(\hat{S}-m T) .
$$

On the other hand we have

$$
d S\left[q_{x x}, R ; m\right)=\int_{\Sigma}\left(\frac{\delta S}{\delta q_{x x}} d q_{x x}+\frac{\delta S}{\delta R} d R\right)+\frac{\partial S}{\partial m} d m=\Theta_{L}+\frac{\partial S}{\partial m} d m
$$


Comparing the last two equations we can identify $\hat{S}-m T=S$ and so we have

$$
T=\frac{\partial S}{\partial m}
$$

This important relation can be verified by an explicit calculation!

We now come to the discussion of configurations for which the observable $T$ does not vanish. Our approach is to start with given values of the observables $T$ and $m$ and ask for those values of $N^{x}$ and $\underset{\sim}{\sim}$ which are compatible with them and with the remaining gauge degrees of freedom:

We begin with the special gauge $E^{1}=x^{2}$ so that $\delta E^{1}=0$ ! Eq. (2.20) implies the condition

$$
N^{x}+\frac{1}{2} x^{2}\left(1-\frac{2 m}{x}\right) \lambda \underset{\sim}{\sim}=0
$$

for $N^{x}$. It shows that $N^{x}$ has to be nonvanishing for a nonvanishing $\lambda$ !

The usual choice for the Schwarzschild parametrization is $N^{x}=0$ so that $\lambda=0$ and $T=0$ in that case.

We see that a nonvanishing $T$ is associated with a slicing (foliation) of spacetime which necessarily has a nonvanishing lapse $N^{x}$ !

Next we ask what gauge freedom is left in general for the gauge dependent functions $N^{x}$ and $\underset{\sim}{N}$ once the observables $m$ and $T$ are given:

In order to answer this question it is convenient to introduce new canonical variables again by defining

$$
s=\ln \left(-B^{1}\right), B^{1}=-e^{s} .
$$

We then have

$$
\pi_{\gamma} d \gamma+\pi_{1} d B^{1}+G d \alpha=i \pi_{\rho} d \rho+\pi_{s} d s+\pi_{\alpha} d \alpha
$$

where

$$
\pi_{\rho}=\pi_{\gamma}\left(B^{1}\right)^{2}, \pi_{s}=2 \pi_{\gamma} \gamma+\pi_{1} B^{1}, \pi_{\alpha}=G,
$$

so that the constraints (see eqs. (4.11) and (4.20)) now take the special form

$$
\pi_{\rho}^{\prime}=0, \pi_{s}=0, \pi_{\alpha}=0 \text {. }
$$

If we define the constraint functional

$$
F\left[\pi_{\rho}, \pi_{s}, \pi_{\alpha}\right]=-\int_{\Sigma}\left(\lambda_{\rho} \pi_{\rho}^{\prime}+i \lambda_{s} \pi_{s}+i \lambda_{\alpha} \pi_{\alpha}\right),
$$


we get from

$\left\{\rho(x), \pi_{\rho}(y)\right\}=\delta(x, y),\left\{s(x), \pi_{s}(y)\right\}=i \delta(x, y),\{\alpha(x), G(y)\}=i \delta(x, y)$ etc.

that

$$
\{\rho(x), F\}=\lambda_{\rho}^{\prime}(x),\{s(x), F\}=\lambda_{s}(x),\{\alpha(x), F\}=\lambda_{\alpha}(x) .
$$

Thus $F$ generates pure gauge transformations: $F$ acts additively on the $O(2)$ angle $\alpha$ and the variable $s$ (i.e. it acts as a scale transformation on $B^{1}$ ). Furthermore, if we put $\rho=\bar{f}^{\prime}$ then $F$ acts additively on $\bar{f}$. All the gauge freedom left over is now contained in the choice of the variables $\alpha, s$ and $\bar{f}$. The choice of $\bar{f}$ is, however, not arbitrary because $8 m \int_{\Sigma} \rho=T$. We therefore introduce

$$
\rho=\frac{T}{8 m} f^{\prime}, \int_{\partial \Sigma} f=1 \text {, i.e. } \lim _{x \rightarrow \infty} f=1 .
$$

This is compatible with the property $\rho \rightarrow O\left(1 / x^{2}\right)$.

Expressed in terms of the observables $m$ and $T$ and the gauge quantities $s$ and $f^{\prime}$ the metric coefficients take the form

$$
\begin{aligned}
q_{x x} & =\frac{\left(R^{\prime}\right)^{2}}{\left(1-\frac{2 m}{R}\right)}-\frac{1}{4} \lambda^{2}\left(1-\frac{2 m}{R}\right) \\
& =4 m^{2} \frac{\left(s^{\prime} e-s\right)^{2}}{\left(1-e^{s}\right)}-\frac{1}{4}\left(T f^{\prime}\right)^{2}\left(1-e^{s}\right), \\
q_{\theta \theta} & =4 m^{2} e^{-2 s} .
\end{aligned}
$$

For the special gauge $q_{\theta \theta}=x^{2}$ we have $\exp (-s)=x /(2 m)$ and

$$
\begin{aligned}
q_{x x} & =\frac{1}{1-\frac{2 m}{x}}-\frac{1}{4}\left(T f^{\prime}\right)^{2}\left(1-\frac{2 m}{x}\right), \\
q_{\theta \theta} & =x^{2} .
\end{aligned}
$$

We finally have to express the shift $N^{x}$ and the "lapse" $\underset{\sim}{\sim}$ in terms of $s$ and $f$ : Observing that $B^{1}=(A / 2-1)$ we get from the eqs. (2.18) and (2.19)

$$
\dot{B}^{1}=\left(B^{1}\right)^{\prime}+m\left(1+B^{1}\right) \lambda \underset{\sim}{\sim}
$$


so that

$$
s^{\prime} N^{x}+m\left(1-e^{-s}\right) T f^{\prime} \underset{\sim}{\sim}=\dot{s} .
$$

A second equation for $N^{x}$ and $\underset{\sim}{\sim}$ we obtain as follows: Eqs. (4.14) and (4.26) yield

$$
\dot{\lambda}=\frac{d}{d x}\left(\lambda N^{x}+\frac{32 m^{3}\left(B^{1}\right)^{\prime}}{A\left(B^{1}\right)^{4}} \stackrel{\sim}{\sim}\right) .
$$

Observing that $\lambda=T f^{\prime}, \dot{T}=2 N^{(\infty)}$ and integrating with respect to $x$ we obtain

$$
T f^{\prime} N^{x}-16 m^{3} \frac{s^{\prime} e^{-3 s}}{\left(1-e^{s}\right)} \stackrel{\sim}{\sim}=2 N^{(\infty)} f+T \dot{f},
$$

where the "constant" of integration $c(t)=0$, due to the asymptotic properties of the different quantities for large $x: f \rightarrow 1, \dot{f} \rightarrow 0, B^{1} \rightarrow-2 m / x, \underset{\sim}{N} \rightarrow$ $1 / x^{2}, N^{x} \rightarrow O\left(1 / x^{2}\right)$.

For the special gauge $q_{\theta \theta}=x^{2}$ we get

$$
\begin{aligned}
x^{2} \underset{\sim}{\sim} & =\frac{2 N^{(\infty)} f+T \dot{f}}{2 q_{x x}} \\
\text { or } N & =\frac{2 N^{(\infty)} f+T \dot{f}}{2 \sqrt{q_{x x}}}, \\
N^{x} & =-\frac{(1-2 m / x) T f^{\prime}\left(2 N^{(\infty)} f+T \dot{f}\right)}{2 q_{x x}},
\end{aligned}
$$

where eqs. (2.10) and (5.29) have been used.

\section{Quantization}

As we have only two physical degrees of freedom, $M$ and $T$, quantization is easy. It depends, however, on the spectrum of the Mass operator $\hat{M}$, namely whether it is bounded from below or not:

1. $-\infty<m<+\infty$.

In this case we have the commutation relations

$$
[\hat{M}, \hat{T}]=-i
$$


Choosing the representation

$$
\hat{T} \phi(T)=T \phi(T), \hat{M} \phi(T)=-i \frac{d}{d T} \phi(T)
$$

with the scalar product

$$
\left(\phi_{1}, \phi_{2}\right)=\int_{-\infty}^{+\infty} d T \phi_{1}^{*}(T) \phi_{2}(T)
$$

we get from eq. (4.32) the Schroedinger eq. for the wave function $\psi(T, t)$ :

$$
i \partial_{t} \psi(T, t)=H \psi(T, t)=2 N^{(\infty)} \hat{M} \psi(T, t)=-i 2 N^{(\infty)} \frac{\partial}{\partial T} \psi(T, t),
$$

which has the solutions

$$
e_{m}(T, t)=\frac{1}{\sqrt{\pi}} e^{-i m(2 \tau(t)-T)}, \quad \text { where } \dot{\tau}=N^{(\infty)}(t)
$$

However, in view of the positive energy theorem for isolated gravitational systems 13 the assumption that the spectrum of $\hat{M}$ is unbounded from below appears to be unphysical and should be changed. We therefore assume:

2. $0<m<\infty$.

Here, if the commutation relation (6.1) still holds, $\hat{T}$ cannot be selfadjoint and therefore not diagonalizable - because otherwise $\exp (i \mu \hat{T}), \mu$ real, would be a unitary operator which generates translations by $\mu$ in $m$-space violating the spectral condition $m>0$. The problem has been discussed in detail by Klauder et al.[14] and by Isham et al. [15]:

If one defines the operator

$$
\hat{S}=\frac{1}{2}(\hat{M} \hat{T}+\hat{T} \hat{M})
$$

then we get the Lie algebra commutator

$$
[\hat{S}, \hat{M}]=i \hat{M}
$$

of the affine group in one dimensions, i.e. $\hat{S}$ generates scale transformations of the spectrum of $\hat{M}$ :

$$
e^{-i \beta \hat{S}} \hat{M} e^{i \beta \hat{S}}=e^{\beta} \hat{M}, \beta \text { real }
$$


In the space of functions $\chi(m)$ we may choose the operator representations

$$
\hat{M}=m, \hat{S}=\frac{i}{2}\left(m \frac{d}{d m}+\frac{d}{d m} m\right)=i\left(m \frac{d}{d m}+\frac{1}{2}\right)
$$

which are selfadjoint with respect to the scalar product

$$
\left(\chi_{1}, \chi_{2}\right)=\int_{0}^{+\infty} d m \chi_{1}^{*}(m) \chi_{2}(m)
$$

and where

$$
\left(e^{i \beta \hat{S}} \chi\right)(m)=e^{-\frac{1}{2} \beta} \chi\left(e^{-\beta} m\right)
$$

leaves the scalar product invariant.

The Schoedinger eq. here has the form

$$
i \partial_{t} \chi(m, t)=2 N^{(\infty)} m \chi(m, t),
$$

with the solutions

$$
\chi(m, t)=e^{-2 i m \tau(t)} g(m),(g, g)<\infty .
$$

The operator $\hat{S}$ has the eigenfunctions

$$
f_{s}(m)=\frac{1}{\sqrt{2 \pi}} m^{-\left(i s+\frac{1}{2}\right)},\left(f_{s_{1}}, f_{s_{2}}\right)=\delta\left(s_{1}-s_{2}\right)
$$

where $s$ is a real eigenvalue of $\hat{S}$.

A more general choice for the scalar product $\left(\chi_{1}, \chi_{2}\right)$ and the operator $\hat{S}$ is

$$
\left(\chi_{1}, \chi_{2}\right)=\int_{0}^{+\infty} d m m^{-\sigma} \chi_{1}^{*}(m) \chi_{2}(m), \hat{S}=i(m d / d m+(1-\sigma) / 2), \sigma \text { real }
$$

As to the unitary representations of the affine group in 1 dimension see ref. [16].

It it easy to verify that the functional $T\left[\pi_{1}, \pi_{\gamma}\right]$ provides the solution of Dirac's algebraic quantization approach, too.

First we observe that the constraint functions $K_{1}(x)$ and $K_{2}(x)$ in eqs. (4.11) 
and (4.20) are first class: Let $\chi_{i}(x), i=1,2$ be 2 suitable test functions. Then it follows from the Poisson brackets (4.31) that

$$
\begin{aligned}
\left\{K_{1}\left(\chi_{1}\right), K_{2}\left(\chi_{2}\right)\right\} & =\int_{\Sigma} d y \int_{\Sigma} d x \chi_{1}(x) \chi_{2}(y)\left\{K_{1}(x), K_{2}(y)\right\} \\
& =-i \int_{\Sigma} d x \chi_{1}(x) \chi_{2}(x) K_{1}(x)=-i K_{1}\left(\chi_{1} \chi_{2}\right)
\end{aligned}
$$

Notice that the constraint functionals $K_{1}\left(\chi_{1}\right)$ and $K_{2}\left(\chi_{2}\right)$ generate the Lie algebra of the affine group in one dimension, too: $K_{2}$ generates a scale transformation of $K_{1}$.

From the Poisson brackets (4.31) we infer the (equal time) operator commutation relations

$\left[\hat{\pi}_{1}(x), \hat{B}^{1}(y)\right]=\delta(x, y),\left[\hat{\pi}_{\gamma}(x), \hat{\gamma}(y)\right]=\delta(x, y),[\hat{G}(x), \hat{\alpha}(y)]=\delta(x, y)$, etc.,

which can be implemented by the choice

$$
\hat{B}^{1}=-\frac{\delta}{\delta \pi_{1}}, \hat{\gamma}=-\frac{\delta}{\delta \pi_{\gamma}}, \hat{\alpha}=\alpha, \hat{\pi}_{1}=\pi_{1}, \hat{\pi}_{\gamma}=\pi_{\gamma}, \hat{G}=\frac{\delta}{\delta \alpha} .
$$

Thus the operator versions of the constraint functions (4.11) and (4.20) take the form

$$
\hat{K}_{1}=-\frac{1}{2} \pi_{\gamma}^{\prime} \frac{\delta}{\delta \pi_{1}}-\pi_{\gamma}\left(\frac{\delta}{\delta \pi_{1}}\right)^{\prime}, \hat{K}_{2}=-2 \pi_{\gamma} \frac{\delta}{\delta \pi_{\gamma}}-\pi_{1} \frac{\delta}{\delta \pi_{1}},
$$

where a suitable choice for the operator-ordering in $\hat{K}_{2}$ has been made. It is easy to see that $\hat{G} T=0, \hat{K}_{1} T=0, \hat{K}_{2} T=0$ so that any complex function $\psi(T)$ is annihilated, too. The first class property (6.16) holds also for the operators $\hat{K}_{1}$ and $\hat{K}_{2}$.

Acknowledgements

We thank P. Hajicek, H. Nicolai and H.-J. Matschull for stimulating discussions. One of us (T.T.) is especially indebted to A. Ashtekar and L. Smolin for many helpful discussions and for their very kind hospitality during several months at the Physics Department of Syracuse University and at the Center for Gravitational Physics and Geometry at Pennsylvania State University. He is also grateful for a Graduierten-Stipendium of the Deutsche Forschungsgemeinschaft and for the associated travel funds. 


\section{References}

[1] T. Thiemann and H.A. Kastrup, Nucl. Phys. B399(1993)211.

This paper contains further references with regard to Ashtekar's work.

[2] S.W. Hawking and G.F.R. Ellis, The large scale structure of space-time (Cambridge University Press, Cambridge, 1973) App. B

[3] A. Ashtekar and J. Samuel, Class. Quantum Grav. 8(1991)2191

[4] E. Witten, Nucl. Phys. B311(1988)46

[5] T. Regge and C. Teitelboim, Ann. Phys. 88(1974)286

[6] R. Beig and N. ó Murchadha, Ann. Phys. 174(1987)463

[7] C.W. Misner, K.S Thorne and J.A. Wheeler, Gravitation (W.H. Freeman and Co. San Francisco 1973) ch. 31

[8] N. Woodhouse, Geometric Quantization (Clarendon Press, Oxford 1980)

[9] E.T. Newman and C. Rovelli, Phys.Rev.Lett. 69(1992)1300

[10] T. Thiemann, PhD Thesis RWTH Aachen, Dec. 1993, and paper to be publ. .

[11] B.K. Berger, D.M. Chitre, V.E. Moncrief and Y. Nutku, Phys.Rev. D5(1972)2467,

F. Lund, Phys.Rev. D8(1973)3247,

R. Benguria, P. Cordero and C. Teitelboim, Nucl.Phys. B122(1977)61

[12] W. Fischler, D. Morgan and J. Polchinski, Phys.Rev. D42(1990)4042

[13] See the review by Y. Choquet-Bruhat, in: Relativity, Groups and Topology II, ed. by B.S. DeWitt and R. Stora, (North Holland, Amsterdam 1984)739

[14] J.R. Klauder and E.W. Aslaksen, Phys. Rev. D 2(1970)272 and refs. therein 
[15] C.J. Isham and A.C. Kakas, Class. Quantum Grav. 1 (1984)621 and 633 ;

C.J. Isham in: Relativity, Groups and Topology II, ed. by B.S. DeWitt and R. Stora, (North Holland, Amsterdam, 1984)1059

[16] E.W. Aslaksen and J.R. Klauder, J. Math. Phys. 9(1968)206 